\title{
La Teoría General de Keynes y la macroeconomía moderna
}

JAIMe Ros*

\section{INTRODUCCIÓN}

La crisis económica mundial de los últimos años es una crisis de gran envergadura que tendrá probablemente importantes repercusiones en años por venir en la teoría macroeconómica y su enseñanza, especialmente a nivel de posgrado. Durante esta crisis, los debates de política macroeconómica de los años 1930, cuando la macroeconomía nació como disciplina con la publicación de la Teoría General de la ocupación, el interés y el dinero (1936) de Keynes, se han repetido nuevamente. Ello se debe, en parte, a la vigencia de la obra de Keynes que tiene por lo menos dos razones de ser. La primera es que la

Artículo recibido en septiembre de 2011; aceptado en enero de 2012.

* Facultad de Economía de la Universidad Nacional Autónoma de México (UNAm), <ros.1@nd.edu>. Una versión preliminar de este artículo fue presentada en septiembre de 2011 en la Conferencia de celebración del 70 Aniversario de la Revista Investigación Económica, en la sesión dedicada al 75 aniversario de la publicación de la Teoría General de Keynes. Agradezco a Ignacio Perrotini, DirectorEditor de Investigación Económica; Anthony P. Thirlwall; Santiago Capraro, mi ayudante de docencia; mis estudiantes de posgrado de Teoría Macroeconómica I, y los participantes en la Conferencia de Investigación Económica que, de diversas maneras, contribuyeron a mejorar la versión presentada en septiembre pasado. Las traducciones al español de las citas originales en inglés son, al igual que otros errores que pueda contener este artículo, de la exclusiva responsabilidad del autor. 
Teoría General es una obra muy moderna en el terreno de los hechos debido a que la actual crisis económica y financiera mundial ha vuelto a poner a los mercados financieros en el epicentro del mal funcionamiento del sistema económico en su conjunto. Y este es precisamente uno de los temas centrales de la Teoría General. La segunda es que esta obra es muy relevante en el terreno de las ideas, tanto para el presente como para el futuro, debido a la vigencia de la crítica de Keynes a lo que llamó la economía clásica. Y lo es, como argumentaré en este artículo, porque si sus críticas a los clásicos son válidas, éstas son igualmente aplicables a la moderna nueva economía clásica.

Este artículo contrasta precisamente las dos visiones de la macroeconomía que se han enfrentado entre sí desde la publicación de la Teoría General y los debates entre su autor y sus críticos de esos años (los clásicos). ${ }^{1}$ Esta comparación hace hincapié en los dos principales temas que separan a estas dos visiones. Las dos primeras secciones del artículo abordan esos temas: la crítica de Keynes a los supuestos de la economía clásica sobre el equilibrio continuo en los mercados y su crítica a los supuestos que esa economía hace sobre el conocimiento que los agentes económicos tienen del futuro. ${ }^{2}$

${ }^{1}$ En mi opinión, la interpretación de la Teoría General que más ilumina las diferencias entre Keynes y sus críticos es la que ofreció, hace ya muchos años, Axel Leijonhufvud en On Keynesian Economics and the Economics of Keynes, publicado en 1968, un libro basado en su tesis de doctorado y que, junto con las contribuciones asociadas de Clower y Bénassy, es seguramente una de las bases necesarias para reconstruir la macroeconomía después de la debacle producida por la contrarrevolución.

2 En la visión clásica que Keynes criticó - representada hoy en día por la nueva economía clásica- los mercados están en equilibrio continuo y los agentes económicos, además de optimizar intertemporalmente, tienen ya sea previsión perfecta o bien, con algunas adecuaciones y en terminología moderna, 'expectativas racionales'. El modelo subyacente, sin formalizar aún cuando Keynes escribió, tiene su origen en un modelo walrasiano dinámico, tal y como fue ampliado y elaborado por Arrow y Debreu. Es interesante observar que ni Arrow ni Debreu pensaban que ese modelo fuera una descripción de la realidad, como muchos en las siguientes generaciones de walrasianos parecen haber creído. Frank Hahn, que hizo importantes contribuciones a la teoría del equilibrio general competitivo, ofrece la mejor descripción que conozco de la función que puede cumplir ese modelo: “[...] el modelo de Arrow y Debreu [...] mostró que es lógicamente posible describir un mundo en el que individuos egoístas y racionales respondiendo sólo a señales de precios toman acciones que son mutualmente compatibles. La teoría no describe a la mano invisible en movimiento sino con la tarea cumplida. La importancia de este logro intelectual es que provee un punto de referencia. Por ello, quiero decir que realiza una función similar a la que un cuerpo ideal y perfectamente saludable realiza para un patólogo clínico cuando mira a un cuerpo real” (Hahn, 1984, p. 308). 
La tercera sección junta estos dos temas y resume lo que me parecen los mensajes de la Teoría General más relevantes para la situación que vive la economía mundial hoy en día: la idea que el desempleo involuntario tiene su origen en una falla de coordinación en los mercados financieros y la idea que la flexibilidad salarial no es una solución a esa falla de coordinación. Las dos últimas secciones comentan brevemente sobre cómo evolucionó la macroeconomía después de la Teoría General, contrastando la economía de Keynes con la de la síntesis neoclásica, la nueva economía clásica y la nueva economía keynesiana.

\section{EL EQUILIBRIO CONTINUO EN LOS MERCADOS, EL RECHAZO DEL SEGUNDO POSTULADO DE LA ECONOMÍA CLÁSICA Y EL DERRUMBE DE LA LEY DE SAY}

¿Cuáles fueron las críticas de Keynes a los clásicos? En primer lugar, Keynes consideraba que la economía clásica descansaba en dos postulados: 1) la igualdad entre el salario real y el producto marginal del trabajo, y 2) la igualdad entre el salario real y la desutilidad marginal del trabajo.

Keynes aceptó el primer postulado. Este proviene simplemente de la condición de primer orden de la maximización de beneficios por parte de empresas que operan en competencia atomística y que, en consecuencia, toman como dados precios y salarios y maximizan, sujetas a una restricción tecnológica (dada por la función de producción). Esta historia de empresas atomísticas que toman los precios como dados, claramente no es cierta, al menos en la gran mayoría de los mercados. Pero Keynes, por razones que creo entender, decidió no meterse con este postulado aunque con seguridad sabía muy bien que la competencia perfecta no era una buena descripción de la situación que prevalece en los mercados de bienes, con excepción de algunos de ellos. El artículo de Sraffa de 1926, la tesis de licenciatura de Kahn a principios de los años 1930 y el libro de Joan Robinson de 1933 versaban sobre la teoría de la competencia imperfecta. Creo por ello, que la decisión de Keynes de aceptar el primer postulado fue una decisión estratégica: le permitió concentrarse en el segundo postulado, su rechazo y las consecuencias de ese rechazo. 
¿De dónde proviene el segundo postulado de la economía clásica? Proviene de las condiciones de primer orden del programa de maximización de la utilidad por parte de hogares trabajadores. Estos hogares maximizan su utilidad, que depende positivamente del consumo y negativamente del trabajo, y, sujetos a una restricción de presupuesto y nada más, maximizan tomando precios y salarios como dados (ya que también son atomísticos). Así, el hogar decide trabajar hasta el punto en que el salario que el trabajador recibe (medido en términos de los bienes que consume) le compensa exactamente por la desutilidad marginal del trabajo. Ni una hora más ni una menos. El resto del tiempo lo dedica al ocio. ${ }^{3}$

Keynes, en los años veinte y treinta del siglo pasado, observaba que, con mucha frecuencia y a veces masivamente, los hogares trabajadores no podían decidir cuánto trabajar, sobre todo cuando estaban desempleados. Es por ello que rechazó el segundo postulado de la economía clásica y en su lugar supuso que el hogar, además de la restricción de presupuesto, enfrentaba una restricción de cantidad respecto al monto de trabajo que podía vender en el mercado. ${ }^{4}$ En suma, lo que Keynes supuso es que los trabajadores no están siempre en sus curvas de oferta de trabajo deseadas; es decir, no siempre pueden vender la cantidad de trabajo que desean.

Al rechazar el segundo postulado de la economía clásica, la ley de Say se derrumba y la demanda agregada pasa a desempeñar un papel clave en la determinación del producto. En efecto, la restricción de cantidad que enfrentan los hogares está dada, para el conjunto de ellos, por la demanda

${ }^{3}$ El segundo postulado puede ilustrarse con los ejemplos de los pequeños agricultores, carpinteros, artesanos o pescadores que, como Robinson Crusoe, poseen sus medios de producción. ¿Pero puede aplicarse también a los asalariados que no tienen posesión de los medios de producción con que trabajan? Es en la respuesta negativa a esta pregunta, es decir en el rechazo al segundo postulado de la economía clásica, que la economía de Keynes y la de Marx se intersectan. Ello explica, creo, porque se intersectan también en el rechazo a la ley de Say. Recuérdese, a este respecto, el "problema de la realización" en Marx y el reconocimiento por parte de Keynes, en el capítulo 3 de la Teoría General, de la contribución de Marx a la teoría de la demanda efectiva junto con las de Malthus, Silvio Gesell y Major Douglas.

${ }^{4}$ Una contribución de Clower (1965), Leijonhufvud (1968) y Bénassy (1975) es haber aclarado precisamente esto. 
de trabajo de las empresas, que puede resultar inferior a la oferta de trabajo deseada por los hogares. Así, ante una caída en el nivel de empleo, de tal magnitud como para generar un exceso de oferta de trabajo, los hogares trabajadores ajustan a la baja su demanda de consumo y el producto total queda determinado por la demanda efectiva. Por cierto, demostrar que al ajustarse a la baja la demanda de consumo, el producto converge de todos modos a un equilibrio estable en el mercado de bienes -con exceso de oferta en el mercado de trabajo- en lugar de continuar descendiendo hasta llegar a cero, debe haber sido un verdadero vía crucis, en el que participó decisivamente Richard Kahn. ${ }^{5}$ Afortunadamente, la condición crucial para la estabilidad de ese equilibrio a menos de pleno empleo es que la propensión marginal a consumir sea menor a la unidad.

Don Patinkin tuvo razón, creo, cuando argumentó que demostrar la estabilidad del equilibrio de menos de pleno empleo es la característica distintiva de la teoría de la demanda efectiva de Keynes. Refiriéndose al famoso diagrama de Paul Samuelson con la recta de 45 grados que intersecta desde abajo una línea de gasto agregado con un intercepto positivo y una pendiente positiva y menor a la unidad, ${ }^{6}$ Patinkin dice: "Lo que llamo la teoría de la demanda efectiva es no sólo que la intersección de la curva de demanda agregada $E=F(Y)$ con la línea de 45 grados determina el producto real de equilibrio Yo a un nivel que puede ser inferior al de pleno empleo [...] sino también (y ésta es la característica distintiva nueva) que el cambio en el producto (y por lo tanto en el ingreso) actúa como una fuerza equilibradora [...] La teoría de la demanda efectiva se refiere no sólo a la solución matemática

${ }^{5}$ Richard Kahn es el economista al que Keynes más agradece, por sus conversaciones, en el prólogo de la Teoría General.

${ }^{6}$ El diagrama de Paul Samuelson es el primer intento por formalizar de una manera sencilla y clara el capítulo 3 de la Teoría General. El modelo IS-LM de Hicks formaliza el modelo de Keynes suponiendo la existencia de dos activos financieros (bonos y dinero) y es en cierta forma más comprehensivo que el diagrama de Samuelson porque contiene un lado real (la curva IS) y un lado financiero (la curva LM). Pero no tiene un mercado de trabajo explícito. El modelo completo de Keynes, con mercado de trabajo y salarios nominales flexibles, se encuentra en el capítulo 19 de la Teoría General. Este modelo completo es muy difícil de formalizar debido a la posible inexistencia de un equilibrio (para una discusión del tema, véase Hahn, 1965). 
de la ecuación de equilibrio $F(Y)=Y$, sino a demostrar la estabilidad de este equilibrio tal y como está determinado por la ecuación de ajuste dinámico $\mathrm{d} Y / \mathrm{d} t=\theta[F(Y)-Y]$, donde $\theta^{\prime}>0$ 0” (1982, p. 9-10).

Esto es lo que explica en qué sentido la teoría propuesta por Keynes se refiere al caso general. En efecto, ¿por qué dice Keynes que su teoría se refiere al caso general del cual la economía clásica es un caso especial? y ¿qué tiene esto que ver con el abandono del segundo postulado de la economía clásica? El caso analizado es el caso general precisamente porque en presencia de un exceso de oferta en el mercado de trabajo, Keynes introduce el supuesto -que debería ser evidente para todo mundo- de que los trabajadores no van a formular sus planes de consumo de la misma forma en que lo hacen cuando el mercado de trabajo está en equilibrio. Si hay exceso de oferta en el mercado de trabajo, los trabajadores enfrentan una restricción de cantidad al monto de trabajo que pueden vender en el mercado, y toman en cuenta esta restricción a la hora de formular su demanda de consumo. Como lo dice Edmond Malinvaud, en un texto que sintetiza las contribuciones de Clower y Bénassy a la comprensión de la economía de Keynes: "Al decidir su demanda de consumo por un bien particular, un individuo desempleado recuerda que está desempleado.” (Malinvaud, 1985, p. 23).

En la economía clásica, y en particular en su expresión más acabada -el modelo walrasiano de equilibrio general-, el trabajador no considera la posibilidad de quedar desempleado a la hora de formular su plan de consumo. La restricción de cantidad no se toma en cuenta en el programa de maximización de su utilidad. Por el contrario, en el plan óptimo que los hogares formulan al maximizar su utilidad, se determinan simultáneamente la demanda de consumo, la cantidad de trabajo y el ocio, suponiendo que no hay restricciones a la cantidad de trabajo que los hogares pueden vender en el mercado. Esto es precisamente lo que dice el segundo postulado de la economía clásica. Pero esto sólo es cierto si el mercado de trabajo despeja porque, si no lo hace, el plan de consumo de los hogares tiene que tomar en cuenta que esos hogares no logran vender la cantidad de trabajo que desean (al salario y precio de los bienes de consumo vigentes).

¿Por qué, desde Walras, los modelos de equilibrio general walrasiano, estáticos o dinámicos hacen caso omiso de la restricción de cantidad, un 
supuesto a todas luces tan extraño? Porque implícitamente o explícitamente, como lo hizo Walras mismo, suponen la existencia de un subastador que tiene, entre otras tareas, la de prohibir que se lleven a cabo transacciones mientras no se haya encontrado el vector de precios de equilibrio que despeja todos los mercados. Y como el trabajador desempleado sabe que el subastador walrasiano no permitirá las transacciones hasta que el mercado de trabajo despeje, con una pureza lógica impecable, formula su plan de consumo suponiendo que puede vender la cantidad de trabajo que desea, sin más restricciones que la de presupuesto, estática o intertemporal, dados los precios y salarios vigentes en el mercado.

\section{EXPECTATIVAS SOBRE EL FUTURO, PREVISIÓN PERFECTA, E INCERTIDUMBRE}

Keynes también rechazó los supuestos de la economía clásica sobre información y previsión del futuro. Como lo dice en su artículo del Quarterly Journal of Economics de 1937 (p. 222): "La teoría ortodoxa supone que tenemos un conocimiento del futuro de una naturaleza muy distinta a la que en realidad poseemos [...] La hipótesis de un futuro calculable conduce a una interpretación incorrecta de los principios de comportamiento que la necesidad de la acción nos obliga a adoptar, y a una subestimación de los factores escondidos de la duda absoluta, la precariedad, la esperanza y el miedo".

En lugar del supuesto de previsión perfecta, Keynes tomó, con frecuencia, las expectativas sobre periodos futuros (o lo que llamó el "estado de las expectativas de largo plazo") como dadas. Las conclusiones del análisis dependen entonces de que las expectativas no cambien ante un cambio supuesto en otras variables: dado el estado de las expectativas (o de las noticias), tal o cual cosa puede suceder como resultado de un cambio en

\footnotetext{
7 Porqué Walras hizo ese supuesto es un tema que está fuera del alcance de este artículo pero tiene que ver con la necesidad de no hacer su tarea imposible ya que el consideró un modelo con $n$ bienes y $\mathrm{m}$ factores de producción, modelo que se vuelve analíticamente muy difícil si se suponen transacciones fuera del equilibrio.
} 
tal o cual variable. En este caso, Keynes sustituyó el supuesto de previsión perfecta por el supuesto de expectativas exógenas que puede atribuirse, como lo plantea Phelps (1990, cap. 1), a la existencia de una comunicación intertemporal incompleta entre participantes del mercado y el problema consiguiente de coordinación de las decisiones intertemporales de ahorro e inversión. ${ }^{8}$

En otras ocasiones, Keynes endogeneiza las expectativas, pero de una manera que toma en cuenta el hecho de que los agentes ignoran el futuro (véase en particular su artículo en el Quarterly Journal of Economics ya citado). $\mathrm{El}$ hecho de que el futuro es desconocido tiene varias consecuencias. La primera es que en nuestras decisiones le damos poco peso a lo que puede suceder en el futuro lejano. Ello se debe a que entre más lejano es el periodo futuro considerado, menos sabemos sobre él y menos podemos aplicar cálculos probabilísticos. Podemos tener una noción (subjetiva) de la distribución de probabilidades de los eventos en el futuro cercano. Entre más lejano el futuro, menos pensamos que sabemos sobre la distribución de probabilidades. Además, sabemos que sabremos más acerca de este periodo futuro a medida que pase el tiempo, de manera que podemos posponer nuestra decisión. Una segunda consecuencia es que tendemos a usar el presente y el pasado reciente como una guía para adivinar el futuro (a menos que tengamos buenas razones para no hacerlo), a pesar de lo endeble que, como dice Keynes (1936), resulta este procedimiento. Un tercer efecto de la incertidumbre es que dependemos de las opiniones de otros (de la opinión promedio) para formular nuestra mejor opinión sobre el futuro. El llamado "concurso de belleza" al que se refiere Keynes es el mejor ejemplo de este comportamiento.

\footnotetext{
${ }^{8}$ Estas decisiones de inversión y ahorro son intertemporales en el sentido preciso de que dependen crucialmente de las expectativas sobre el futuro. Las empresas invierten hoy, no para producir hoy, sino para producir en el futuro. El ahorro también es una decisión intertemporal en el sentido de que los hogares ahorran hoy para consumir en el futuro (o simplemente para acumular riqueza futura). En ambos casos, las expectativas sobre las condiciones futuras cumplen un papel crucial en esas decisiones.
} 
Otra observación aguda que aparece en la Teoría General de múltiples maneras -en la discusión de la función consumo, los efectos de cambios en los salarios, etcétera- es que el efecto en las expectativas sobre el futuro de un cambio en una variable económica depende de cómo este cambio es percibido por los agentes económicos. Cambios que son percibidos como de corto plazo, reversibles (temporales, en terminología moderna) tendrán poco efecto sobre el valor esperado en el futuro de la variable en cuestión, mientras que cambios que son percibidos como de naturaleza más permanente tendrán un mayor efecto en los valores futuros esperados. La elasticidad de las expectativas depende de estas percepciones y éstas varían de acuerdo con las circunstancias.

\section{LA TEORÍA DEL DESEMPLEO INVOLUNTARIO}

Poner estos dos temas juntos, el del equilibrio en los mercados y el de las expectativas sobre el futuro, conduce a una distinción sutil entre la eficiencia de un mercado en el sentido de si despeja o no y que tan rápido lo hace, y el papel de un mercado como fuente de mal funcionamiento del sistema económico. Por ejemplo, para Keynes el mercado de trabajo no despeja tan rápido como los mercados financieros. Sin embargo, lo que Keynes llama desempleo involuntario tiene su origen en el hecho de que los mercados financieros, aunque despejan todo el tiempo, pueden hacerlo a "precios erróneos": son capaces de despejar a una tasa de interés que es inconsistente con el pleno empleo en el mercado de trabajo. Dicho a la vieja usanza, la tasa de interés de equilibrio puede ser demasiado alta para que la inversión deseada por las empresas a esa tasa de interés logre absorber los ahorros correspondientes al nivel de ingreso de pleno empleo. El nivel de ingreso, y no la tasa de interés, se ajusta entonces a la baja y con ello el nivel de ahorro queda por debajo del nivel de pleno empleo.

En esta visión los mercados financieros son los principales candidatos para caer en fallas de información (especulación mal informada) precisamente porque están encargados de la coordinación de las decisiones de producción y consumo en el futuro. El desempleo generado por estas fallas de 
información sobre el futuro, y de las resultantes fallas de coordinación de las decisiones de ahorro e inversión, es involuntario precisamente en el sentido de que no tiene su origen en el funcionamiento del mercado de trabajo sino en el mal funcionamiento de los mercados financieros. Y ello no es culpa de nadie. Es el resultado de que la coordinación de las decisiones intertemporales se lleva a cabo en condiciones de incertidumbre (o, si se prefiere, de ignorancia) sobre el futuro. Como lo dice Leijonhufvud: "En el mundo keynesiano, los mercados financieros son manifiestamente incapaces de proveer la consistencia de los planes de producción y consumo de largo plazo" (Leijonhufvud, 1968, p. 276).

El desempleo es involuntario también en el sentido de que la flexibilidad a la baja de los salarios nominales no es una solución a este problema de coordinación entre decisiones de ahorro e inversión, como Keynes lo argumenta en el capítulo 19 de la Teoría General. Volveremos a este tema en la siguiente sección pero vale la pena enfatizar que varios keynesianos, de Kalecki (1944) a Leijonhufvud (1968), pasando por Patinkin (1965) y Tobin (1980), y otros artículos y conferencias), entendieron muy claramente el argumento de Keynes y sus implicaciones de política económica. Creo que Tobin es quien lo dijo mejor: "(Keynes) reta a la ortodoxia en terreno sagrado, su fe en que la competencia ajustará los precios de los bienes y factores de manera a eliminar excesos de oferta, o de demanda, en todos los mercados. No dice meramente que este proceso puede tomar un tiempo muy largo; dice que simplemente no funciona [...] la moraleja práctica es que una política activa, junto con respuestas de mercado, es parte del mecanismo social para el mantenimiento o restablecimiento del equilibrio" (Tobin, 1980, p. 2).

Hay aquí un gran contraste con la macroeconomía moderna. En la nueva economía clásica, la explicación de los ciclos económicos también considera la existencia de información incompleta (como en el caso del modelo Lucas-Phelps) pero ubica las fallas de información en el mercado de trabajo (trabajadores que confunden aumentos en los salarios nominales con aumentos en los salarios reales) o en los mercados de bienes (agentes que confunden cambios en el nivel general de precios con cambios en precios relativos). De manera similar, en la nueva economía keynesiana de las últimas 
décadas, el desempleo se explica, en algunas versiones, como resultado de imperfecciones o fallas de información en el mercado de trabajo.

\section{La MaCroeconomía desPués de La TeORÍA GeNerAL}

En cuanto a lo que ocurrió con la macroeconomía keynesiana después de la Teoría General quiero poner el acento en tres temas. El primero se refiere al supuesto de salarios nominales dados (que Keynes mantuvo hasta el capítulo 19 de la Teoría General en que analiza el caso general de flexibilidad de salarios y precios). El segundo se refiere a la pérdida de relevancia en la síntesis neoclásica, que se origina en el modelo IS-LM de Hicks, de las expectativas sobre el futuro en la determinación del nivel actual de actividad económica y empleo. El tercero tiene que ver con la reintroducción del segundo postulado de la economía clásica en la macroeconomía pos Teoría General

\section{El papel del supuesto de salarios nominales dados}

Como lo ha hecho ver Patinkin, para Keynes la rigidez de los salarios nominales es más una recomendación de política económica que un supuesto de comportamiento ¡Cómo podría ser de otra manera si la gran depresión de los años treinta del siglo pasado registró una deflación sin precedente de los salarios nominales! En cambio, en la síntesis neoclásica, y más aún entre los nuevos keynesianos, la rigidez de los salarios nominales (o de los precios en algunas versiones) se convirtió en un supuesto clave para la existencia de un equilibrio con desempleo. Ello abrió las puertas para la resurrección de la principal conclusión de la economía clásica: con precios y salarios plenamente flexibles, la economía tiende al pleno empleo.

El comentario de Kalecki al profesor Pigou (1943), publicado en el Economic Journal de 1944, esta ahí para recordarnos la falla en el razonamiento que conduce a esa conclusión. En ese comentario de dos páginas, Kalecki argumenta que el efecto positivo sobre la demanda agregada del aumento en los saldos monetarios reales, provocado por la deflación, se verá contrarrestado con toda seguridad por el aumento en el valor real de las deudas de 
empresas y familias y su efecto negativo sobre la demanda agregada. Esto es así, argumentó Kalecki, porque en una economía monetaria moderna los saldos monetarios son, en su mayor parte, depósitos bancarios que tienen, como contraparte en la hoja de balance de los bancos, activos de los bancos que son, a su vez, deuda de empresas y hogares.

El argumento de Kalecki implica, en términos del modelo de oferta y demanda agregada de los libros de texto, que la curva de demanda agregada (en espacio nivel de precios, nivel de producto) no tiene porqué tener pendiente negativa. Y, por lo tanto, el desplazamiento hacia abajo de la curva de oferta agregada, resultante de la caída de los salarios nominales, no tiene porqué llevarnos a un equilibrio con pleno empleo. Este es un tema, como ya lo mencioné al final de la sección anterior, que ha sido enfatizado también por otros keynesianos.

\section{Las expectativas sobre el futuro en la síntesis neoclásica}

Como lo ha argumentado Leijonhufvud (1968), un tema central de la Teoría General es cómo opiniones cambiantes sobre el futuro son capaces de influir en el nivel actual de ocupación. El vínculo crucial es la inversión que afecta a los niveles presentes de actividad económica y de empleo y que, como ya lo mencioné, es una decisión intertemporal que depende de las expectativas sobre el futuro. Es interesante observar que la ausencia de las expectativas sobre el futuro en la determinación del nivel de ingreso de equilibrio inferior al de pleno empleo es la principal objeción de Keynes al modelo IS-LM de Hicks. En efecto, en la correspondencia entre Keynes y Hicks después de que Hicks formulara su modelo IS-LM en "Mr. Keynes and the classics" (1937), Keynes le escribe a Hicks que tiene poco que agregar a su formalización excepto porque la función de inversión (en la que Hicks hace depender la inversión del nivel de ingreso presente) debería depender de condiciones futuras (porque las empresas invierten, no para producir en el presente, sino para producir en el futuro). Es interesante también que esto es prácticamente todo lo Keynes tiene que agregar a la formulación de Hicks!

Ese tema central se perdió en la síntesis neoclásica, lo que abrió las puertas para la reemergencia de modelos de optimización intertemporal en 
la nueva economía clásica (y también entre los nuevos keynesianos) que, en cierto modo, echaron fuera de la bañera al bebé junto con el agua sucia: es decir, reintrodujeron correctamente la importancia de las expectativas sobre el futuro -en la que la Teoría General había hecho tanto hincapié-, pero descartaron los necesarios supuestos sobre la incertidumbre fundamental que tienen los agentes económicos acerca del futuro.

\section{La reintroducción del segundo postulado de la economía clásica y con ello del supuesto de equilibrio continuo en el mercado de trabajo}

Las puertas para que avanzara la contrarrevolución se abrieron de par en par con la Presidential Address de Milton Friedman a la American Economic Association en 1968 ("El papel de la política monetaria”). Esa ponencia es un hito porque marca, hasta donde puedo ver, el momento en el que, en la macroeconomía post Teoría General se reintroduce el supuesto que el mercado de trabajo despeja en todo momento, es decir que el mercado de trabajo está en equilibrio continuo. En efecto, el modelo implícito de Friedman en esa ponencia supone que ese mercado siempre está en equilibrio y los efectos de la demanda agregada sobre el nivel de actividad, provocados por choques monetarios, se dan como resultado de fluctuaciones en la oferta de trabajo, sin que ello implique cambios en el nivel de desempleo. Estas fluctuaciones en la oferta de trabajo son el resultado de que los trabajadores confunden temporalmente los aumentos en salarios nominales con aumentos en salarios reales (lo que conduce a un aumento de su oferta de trabajo) al no darse cuenta de inmediato que los precios, al igual que los salarios nominales, también aumentan con la expansión monetaria. Pero en todo este proceso, el mercado de trabajo está siempre en equilibrio y los trabajadores pueden en todo momento vender la cantidad de trabajo que desean a los precios y salarios que perciben. En otras palabras, una expansión (o contracción) de la demanda agregada no afecta el nivel de actividad reduciendo (o expandiendo) el desempleo: los trabajadores siempre están en su curva de oferta de trabajo deseada. El segundo postulado de la economía clásica se vuelve a cumplir. 
El famoso modelo de Lucas (1972) no es más que una variación sobre el mismo tema. El modelo es esencialmente el de Friedman, con dos diferencias. Primero, en el modelo de Lucas los agentes económicos son en cierta forma pescadores que pescan diferentes tipos de peces, a diferencia del modelo de Friedman en el que los agentes son empresas y trabajadores. Segundo, en el modelo de Lucas los agentes económicos tienen expectativas racionales (en lugar de expectativas adaptativas como en Friedman). Debido a la falta de información completa, los agentes económicos confunden también temporalmente cambios en el nivel general de precios con cambios en los precios relativos, haciendo así que el dinero pueda no ser neutral a corto plazo. Es precisamente por el hecho que ahora las expectativas son racionales y de que hay información incompleta que es necesario hacer una distinción entre los efectos de choques monetarios anticipados -que son neutrales aun en el muy corto plazo- y los de choques monetarios no anticipados que no son neutrales en el corto plazo.

\section{LA EDAD OBSCURA DE LA TEORÍA MACROECONÓMICA}

Desde entonces hemos vivido en lo que Paul Krugman ha llamado "la edad obscura de la macroeconomía” (Krugman, 2011). En estos años, los últimos treinta, piensa Krugman, sucedieron dos cosas. En primer lugar, se destruyó conocimiento previamente acumulado con consecuencias sociales nefastas. En efecto, se repiten hoy, con una fe que no existía hace treinta o cuarenta años, las mismas falacias que se decían en los años treinta antes y después de la publicación de la Teoría General. Para la ortodoxia, por ejemplo, el desempleo involuntario (cuando se admite su existencia, lo cual es raro) vuelve a ser resultado de la rigidez a la baja de los salarios nominales y, más sorprendente y notable aún, se atribuye a Keynes esa explicación del desempleo! Considérese, por ejemplo, la siguiente cita de Gary Becker (2009) de la Universidad de Chicago y premio Nobel de Economía:

Keynes y muchos economistas anteriores a él enfatizaron que el desempleo aumenta durante las recesiones porque las tasas de salario nominal tienden a ser inflexibles a la baja. La forma natural en que los mercados usualmente eliminan 
una demanda insuficiente por un bien o servicio, tal como el trabajo, es por la vía de una reducción del precio de ese bien o servicio. Una caída en el precio estimula la demanda y reduce la oferta hasta que éstas se vuelven aproximadamente iguales entre sí. La inflexibilidad a la baja de los salarios impide que eso ocurra rápidamente cuando hay una demanda insuficiente de trabajadores. ${ }^{9}$

Véase también sobre el mismo tema lo que dice Robert Barro (2009) de la Universidad de Harvard (citado por Krugman, 2011):

John Maynard Keynes sostuvo que el problema radicaba en que los precios y los salarios tenían un nivel excesivamente alto. Sin embargo, ese problema podía solucionarse fácilmente a través de una política monetaria expansiva, de tal forma que los precios y los salarios no tendrían que caer.

Al igual que en los años treinta del siglo pasado, de acuerdo con la ortodoxia un aumento del gasto público necesariamente desplaza un monto igual de gasto privado. En este sentido, Krugman (2011) cita a John Cochrane (2009) de la Universidad de Chicago:

Primero, si no se imprime dinero, éste tiene que venir de algún lugar. Si el gobierno nos pide prestado un dólar, ese dólar no los vamos a gastar o a prestarlo a una empresa para que realice una nueva inversión. Cada dólar en que se incrementa el gasto público debe corresponder a un dólar menos de gasto privado. Los puestos de trabajo creados a través de un gasto estimulador son compensados por pérdidas de empleos causadas por la caída en el gasto privado. Se pueden construir caminos en vez de fábricas, pero el estímulo fiscal no nos puede ayudar a construir más de ambos. Esto es contabilidad, y no necesita un argumento complejo para describir el fenómeno del desplazamiento (crowding out).

Sobre el mismo tema, Krugman (2011) cita también a Niall Ferguson, historiador de la Universidad de Harvard, (en Soros et al., 2009), una cita

\footnotetext{
9 Para un análisis mayor véase: Rajiv Sethi, 2009. On the consequences of nominal wage flexibility. Rajivsethi.blogspot.com Rajiv Sethi. Thoughts on economics, finance, crime and identity..., [blog] 08 de diciembre. Disponible en: < http:/ / rajivsethi.blogspot.com/2009/12/on-consequences-ofnominal-wage.html>.
} 
extraordinaria en un momento en que la tasa de interés de corto plazo había caído ya prácticamente a cero y la base monetaria se había multiplicado varias veces:

Ahora estamos en la fase de terapia ¿y qué terapia estamos siguiendo? Bueno, es una muy interesante porque tiene dos componentes contradictorios. Uno es el prescrito por el Dr. Friedman -es decir, Milton Friedman- que ha sido administrada por la Reserva Federal: inyecciones masivas de liquidez para evitar el tipo de crisis bancaria que causó la Gran Depresión a principios de la década de 1930. Yo estoy de acuerdo con esa. Es correcto hacer eso. Pero hay otro componente de la terapia que es administrado de forma simultánea, que fue prescrito por el Dr. Keynes -John Maynard Keynes- y esta parte de la terapia implica la generación de un déficit que excederá el 12\% del pIB el corriente año y la emisión, por lo tanto de un gran cantidad de nuevos bonos.

Existe una clara contradicción entre estas dos políticas, y estamos tratando de tenerlas a ambas. No se puede ser monetarista y keynesiano al mismo tiempo -por lo menos no sé cómo se puede hacer, porque si el objetivo de una política monetarista es mantener la tasa de interés baja y mantener una liquidez alta, el efecto de la política keynesiana es presionar al alza la tasa de interés.

Después de todo, 175000 millones de dólares es una gran cantidad de nuevos bonos puestos en el mercado de bonos en el momento de la recesión, y todavía no sabemos bien quién los comprará. Ciertamente, no serán los chinos. Eso funcionó bien, en los buenos tiempos, pero lo que yo llamo quimérico, el matrimonio entre China y América, está alcanzando su final. Tal vez termine en un divorcio escandaloso.

Todo esto ayuda a explicar porqué Krugman afirma que si todo lo que supiéramos cuando la actual crisis estalló fuera la macroeconomía de los años 1950 habríamos estado mejor preparados para enfrentarla. Y también porqué Robert Lucas, de la Universidad de Chicago y premio Nobel de Economía, podía decir con confianza, en plena edad obscura, sólo cinco años antes de la crisis reciente lo siguiente:

Mi tesis en esta lección es que la macroeconomía en este sentido original [como un campo distinto dentro de la economía desde los años cuarenta] ha tenido éxito: su problema central de prevención de la depresión ha sido resuelto, para todo propósito práctico, y de hecho ha sido resuelto desde hace muchas décadas (Lucas, 2003, p. 1). 
De manera que un primer aspecto de la edad obscura es la destrucción de conocimiento. El segundo aspecto es que, en estos años, ha predominado una visión de la macroeconomía en la que se supone que los agentes económicos, con gran información sobre el funcionamiento de la economía y basados en las correspondientes expectativas racionales, formulan sus planes óptimos de producción, inversión, consumo, trabajo y ocio, y los mercados, en equilibrio continuo, se encargan de volver consistentes entre sí esos planes óptimos. Otra manera de referirse a esta visión es, en la expresión de Buiter (1980), como la macroeconomía del Dr. Pangloss.

¿Cómo pueden estos modelos walrasianos dinámicos, con equilibrio continuo en todos los mercados, explicar los ciclos económicos, la alternancia en la economía de periodos de auge y empleo alto seguidos de recesiones y alto desempleo, uno de los fenómenos más importantes que dieron origen a la macroeconomía? La moderna nueva economía clásica tiene dos principales respuestas a esta pregunta. Una está basada en la existencia de perturbaciones monetarias (choques de demanda agregada que como en el modelo Lucas-Phelps dan lugar a fluctuaciones del producto en presencia de información imperfecta). Ya comenté sobre el mecanismo que permite que la demanda agregada tenga un efecto sobre la actividad económica. La otra respuesta está basada en perturbaciones reales, en particular choques tecnológicos que, en la literatura sobre ciclos económicos reales, conducen a fluctuaciones del producto a través de varios mecanismos posibles de propagación (entre otros, la sustitución entre trabajo presente y futuro). En este caso, la demanda agregada no cumple ningún papel.

Creo que sobra decir que estas explicaciones del ciclo económico no tienen nada que ver con la crisis actual, la cual, esperemos, se encargará de echarlas $-\mathrm{y}$ junto con ellas al segundo postulado de la economía clásica- al basurero de la historia de la macroeconomía. Y esperemos que, en el caso del segundo postulado de la economía clásica, esta vez sea la definitiva.

\section{REFERENCIAS}

Barro, R., 2009. Government spending is no free lunch. Wall Street Journal, January 22. 
Becker, 2009. How to increase employment. Becker-Posner-blog.com The Becker-Posner blog, [blog]. Disponible en: <http://www.becker-posner-blog. com/2009/11/how-to-increase-employment--becker.html $>$.

Bénassy, J.P., 1975. Neo-Keynesian disequilibrium theory in a monetary economy. The Review of Economic Studies, 42(4), pp. 503-23.

Buiter, W., 1980. The macroeconomics of Dr. Pangloss: a critical survey of the New Classical Macroeconomics. Economic Journal, 90, pp. 34-50.

Clower, R., 1965. The Keynesian counter-revolution: a theoretical appraisal. En: F. Hahn y F. Breching, eds. The theory of Interest Rates. Londres: Macmillan.

Cochrane, J., 2009. Fiscal stimulus, fiscal inflation, or fiscal fallacies? Disponible en: $<$ http:// faculty.chicagobooth.edu/john.cochrane/research/Papers/fiscal2. html>.

Friedman. M., 1968. The role of monetary policy. The American Economic Review, 58 (1), pp. 1-17.

Hahn, F., 1984. Equilibrium and Macroeconomics. Cambridge, MA: The мгт Press, 394.

Hahn, F., 1965. On some problems of proving the existence of equilibrium in a monetary economy. En: F. Hahn and F. Brechling, eds. Theory of Interest Rates. Londres: Macmillan, pp. 126-35.

Hicks, J., 1937. Mr. Keynes and the Classics: a suggested interpretation, Econometrica, $5(2)$.

Kalecki, M., 1944. Professor Pigou on "The Classical Stationary State" a comment. The Economic Journal, 54(213), abril, pp. 131-2.

Keynes, J.M., 1936. The General Theory of Employment, Interest and Money. Nueva York: Harcourt and Brace.

Keynes, J.M., 1937. The general theory of employment, Quarterly Journal of Economics, 51(2), pp. 209-23.

Soros, G. et al., 2009. The crisis and how to deal with it. New York Review of Books, junio 11.

Krugman, P., 2011. Mr. Keynes and The Moderns. En: University of Cambridge, Cambridge Conference Commemorating the 75th Anniversary of the publication of The General Theory of Employment, Interest, and Money. 20-21 de junio de 2011. Cambridge, MA: University of Cambridge.

Leijonhufvud, A., 1968. On Keynesian Economics and the Economics of Keynes: A Study in Monetary Theory, Nueva York: Oxford University Press.

Lucas, R., 1972. Expectations and the neutrality of Money. Journal of Economic Theory, 4, pp. 103-24. 
Lucas, R., 2003. Macroeconomic priorities. [mimeo] Chicago: Department of Economics, The University of Chicago.

Malinvaud, E., 1985. The Theory of Unemployment Reconsidered. Nueva York: B. Blackwell.

Patinkin, D., 1965. Money, Interest, and Prices: an integration of monetary and value theory. Nueva York: Harper \& Row.

Patinkin, D., 1982. Anticipations to the general theory? And other essays on Keynes. Chicago: The University of Chicago Press, 302.

Phelps, E., 1990. Seven Schools of Macroeconomic Thought: the Arne Ryde memorial lectures. Nueva York: Oxford University Press.

Pigou, A.C., 1943. The Classical Stationary State. The Economic Journal, 53(212), pp. 343-51.

Robinson, J., 1933. The Economics of Imperfect Competition. Londres: Macmillan.

Sraffa, P., 1926. The laws of returns under competitive conditions. Economic Journal, 36(144), pp. 535-50.

Tobin, J., 1980. Asset Accumulation and Economic Activity: reflections on contemporary macroeconomic theory. Chicago: University of Chicago Press. 